\title{
Comparative efficacy, safety and durability of dolutegravir relative to common core agents in treatment-naïve patients infected with HIV-1: an update on a systematic review and network meta-analysis
}

\author{
Katharina Nickel ${ }^{1}$, Nicholas J. A. Halfpenny ${ }^{2}$, Sonya J. Snedecor ${ }^{3}$ and Yogesh Suresh Punekar ${ }^{4 *}$ (D)
}

\begin{abstract}
Background: The objective of this study was to assess the durability of response of dolutegravir (DTG) as an antiretroviral core agent by comparing its efficacy and safety with other recommended or commonly used core agents up to 96-weeks (W96).

Methods: A previously published systematic review was updated to identify phase 3/4 randomised controlled trials (RCTs) of core agents in treatment-naïve HIV-1 patients. Efficacy [virologic suppression (VS), CD4 ${ }^{+}$cell change from baseline] and safety [adverse events [AEs], discontinuations, drug-related AEs [DRAEs]] were analysed at W96 using Bayesian network meta-analysis (NMA) adjusting for nucleoside/nucleotide reverse transcriptase inhibitors' (NRTIs') backbone. Subgroups of patients with $\mathrm{VL}>100,000$ copies $/ \mathrm{mL}$ or CD4 ${ }^{+} \leq 200$ cells/ $\mu \mathrm{L}$ at baseline were analysed separately.

Results: The NMA included 20 studies reporting data at W96. A higher proportion of patients receiving DTG achieved VS compared to those on protease inhibitors [PI:Range:8.7\%(Crl:3.1,16.0)-19.9\%(10.8,30.5)], efavirenz [EFV:6.9\%(1.3,10.8)] and cobicistat-boosted elvitegravir [EVG/c:8.2\%(0.2,17.4)], and similar but numerically higher compared to rilpivirine [RPV:5.0\%(2.8,12.5)], raltegravir [RAL:2.9\%(- 1.6,7.7)] and bictegravir [BIC:2.7\%(- 2.7,10.6)]. The probability that more patients on DTG would achieve VS at W96 compared to any other core agent was greater than 80\%. A higher proportion of patients on DTG achieved VS compared to PI/rs [Range:33.1\%(13.6,50.4)-45.3\%(24.1,61.6)] and RAL [16.7\%(3.3,31.2)] in patients with VL > 100, 000 copies $/ \mathrm{mL}$ at baseline, and similar VS was achieved in patients with $\mathrm{CD}^{+} \leq 200$ cells/ $\mu \mathrm{L}$ at baseline. DTG also achieved greater increase in $\mathrm{CD}^{+}$cells from baseline compared to EFV [32.6(10.7,54.7)], ritonavir-boosted darunavir [DRV/r:25.7(3.6, 48.1)] and BIC [24.7(1.5,47.7)]. Patients receiving DTG had lower odds of discontinuing therapy by W96 compared to Pl/rs, EFV, RAL and EVG/c. Patients on DTG had lower odds of experiencing an adverse event (AE) compared to patients on EFV [odds ratio:0.6(0.3,0.9)], ATV/r [0.4(0.3,0.6)] and LPV/r [0.3(0.2,0.5)]. For patients on DTG, the odds of experiencing a drug-related AE were lower than the odds for patients on EFV [0.3(0.2,0.4)], comparable to patients on RAL [1.1 $(0.8,1.4)]$ and higher than (Continued on next page)
\end{abstract}

\footnotetext{
* Correspondence: yogesh.q.punekar@gsk.com

${ }^{4}$ ViiV Healthcare, GSK House, 980 Great West Rd, Brentford, Middlesex TW8

9GS, UK

Full list of author information is available at the end of the article
}

(c) The Author(s). 2021, corrected publication 2021. Open Access This article is licensed under a Creative Commons Attribution 4.0 International License, which permits use, sharing, adaptation, distribution and reproduction in any medium or format, as long as you give appropriate credit to the original author(s) and the source, provide a link to the Creative Commons licence, and indicate if changes were made. The images or other third party material in this article are included in the article's Creative Commons licence, unless indicated otherwise in a credit line to the material. If material is not included in the article's Creative Commons licence and your intended use is not permitted by statutory regulation or exceeds the permitted use, you will need to obtain permission directly from the copyright holder. To view a copy of this licence, visit http://creativecommons.org/ licenses/by/4.0/. The Creative Commons Public Domain Dedication waiver (http://creativecommons.org/publicdomain/zero/1. 0/) applies to the data made available in this article, unless otherwise stated in a credit line to the data. 
(Continued from previous page)

those on BIC [1.5(1.1,2.0)].

Conclusion: Un-boosted integrase inhibitors had better efficacy and similar safety compared to Pl/rs at W96 in treatmentnaive patients with HIV-1, with DTG being among the most efficacious core agent, particularly in patients with baseline VL > 100,000 copies $/ \mathrm{mL}$ or $\leq 200 \mathrm{CD} 4^{+}$cells/ $\mu \mathrm{L}$, who can be difficult to treat.

Keywords: Antiretroviral therapy, Dolutegravir, HIV-1, Network meta-analysis, Systematic review, Treatment-naïve, Integrase strand inhibitors, Non-nucleoside reverse transcriptase inhibitor, Protease inhibitor

\section{Background}

Human Immunodeficiency Virus type 1 (HIV-1) is a retrovirus that can lead to acquired immunodeficiency syndrome (AIDS), an advanced stage of HIV infection wherein the immune system is severely damaged. The advent of a multi-drug antiretroviral therapy (ART) has transformed HIV into a chronic condition with life expectancy comparable to that of the general population [1]. All the major guidelines recommend first-line ART composed of a core agent belonging to the integrase strand transfer inhibitors [INSTI] class in combination with one or two nucleoside/nucleotide reverse transcriptase inhibitors (NRTIs) [2-4]. European AIDS Clinical Society (EACS) guidelines also recommend specific core agents belonging to the ritonavir-boosted protease inhibitor $[\mathrm{PI} / \mathrm{r}]$ class with two NRTIs as preferred regimen and specific core agents belonging to the nonnucleoside reverse transcriptase inhibitor [NNRTI] class with two NRTIs as an alternative regimen [3]. Overall, the most commonly used and recommended core agents include bictegravir (BIC), dolutegravir (DTG), cobicistatboosted elvitegravir $(E V G / c)$, and raltegravir (RAL) belonging to the INSTI class; atazanavir (ATV/r), daruna$\operatorname{vir}(\mathrm{DRV} / \mathrm{r})$, and lopinavir (LPV/r) belonging to the $\mathrm{PI} / \mathrm{r}$ class; or efavirenz (EFV) and rilpivirine (RPV) belonging to the NNRTI class. These core agents, which differ in their efficacy, provide the antiretroviral strength to the combination allowing ARTs to vary in their ability to achieve and maintain virological suppression (VS). Furthermore, all classes of core agents are associated with tolerability and toxicity concerns with significant variations within each class [5]. It is therefore imperative to compare these agents in their efficacy, safety and durability to identify an ART suitable for appropriate patients.

Network meta-analysis (NMAs) allows comparison of individual core agents, based on a network of randomized clinical trial evidence, where head to head data are unavailable. A recent meta-analysis compared these core agents on efficacy outcomes such as VS and CD4+ cell count change from baseline, and safety outcomes including adverse events [AEs], discontinuations, discontinuation due to AEs and lipid changes [6]. Authors concluded INSTIs to have superior efficacy and comparable safety to PIs and NNRTIs with DTG being among the most efficacious INSTI in treatment-naïve HIV-infected patients. This study, however, was restricted to 48-weeks (W48), thus providing no evidence on the durability of these treatment effects. Another meta-analysis conducted in 2016 reported results up to 96-weeks (W96) but did not include newer core agents such as BIC [7]. The objective of our study was to compare DTG against other guideline-recommended core agents in treatment-naïve HIV-infected patients, to update our earlier work using evidence up to W96.

\section{Methods}

A systematic search of PubMed/MEDLINE, Embase, and Cochrane databases was undertaken on July 19, 2019 to update the original search conducted in 2013 and updated in 2018 [6, 8]. Randomised controlled trials (RCTs) evaluating the efficacy and/or safety of ARVs in treatmentnaive people living with HIV (PLHIV) were identified. The search strategy for PubMed and Embase is available upon request. Further searches were conducted in the National Institute of Health clinical trial (NCT) registry database (www.clinicaltrials.gov). Additional records were identified through manual searching of article references. Two independent reviewers screened the study titles/abstracts to select studies which were further screened after reviewing full-text articles. Any discrepancies between the reviewers were resolved by consensus. Study data were extracted into a structured database by at least two independent reviewers and reconciled for accuracy. The Preferred Reporting Items for Systematic Reviews and MetaAnalyses (PRISMA) guidelines were followed in all phases of the study [9].

Studies were included if they were phase $3 / 4$ RCTs of treatment-naïve adults or adolescents ( $\geq 13$ years of age) with HIV-1 infection published in the English language. In addition, all studies were required to compare at least two of the core agents of interest in combination with two NRTIs and report at least one of the efficacy or safety outcomes of interest [6]. Studies investigating various dosage strengths of a core agent without an active comparator, with a sample size of less than 50 patients, or with paediatric populations ( $<13$ years of age) were excluded. The core agents included in the NMA were 
INSTIs (DTG, BIC, EVG/c, RAL), ritonavir-boosted PIs (ATV/r, DRV/r, LPV/r), and NNRTIs (EFV, RPV) [2-4].

The efficacy outcomes included in the NMA were the proportion of patients with VS at W96 and the change from baseline in CD4+ cell count at W96. In accordance with FDA guidance [10], VS was calculated as FDA Snapshot-50, time to loss of virologic response-50 (TLOVR-50), confirmed virologic response-50 (CVR-50), and HIV RNA $<50$ copies $/ \mathrm{mL}$, and utilized within the NMA in that order of preference. The safety outcomes included were the proportion of patients with any $\mathrm{AE}$, overall discontinuations, and drug-related AEs. In addition to the overall population, efficacy and safety outcomes were assessed in subgroups of patients with baseline viral load (VL) $\leq 100,000$ and $>100,000$, and patients with baseline CD4+ cell count $\leq 200$ and $>200$ cells/ $\mu \mathrm{L}$ (secondary objective).

A Bayesian analysis framework was used to generate estimates of efficacy and safety of core agents relative to DTG [11, 12] using WinBUGS (version 1.4.3). Established frameworks were used to construct outcomesbased models [13]. For each outcome, a fixed-effect (FE) model and a random-effect (RE) model were evaluated.
The Deviance Information Criterion (DIC) was used to select the model with a better fit between the FE and RE models. Further analyses were conducted to assess the heterogeneity in the treatment effects and inconsistency in the connected network.

Analyses were adjusted by the NRTI backbone combination included within each regimen. NRTI combinations were grouped into three categories: abacavir/lamivudine (ABC/3TC), tenofovir disoproxil (or alafenamide) fumarate/ emtricitabine (TD[A]F/FTC), or any other NRTI combination (Other). Vague prior distributions (e.g. normal with mean 0 and variance $10^{5}$ ) on model parameters were used so that outcomes would be determined only by data from the RCTs. Posterior outcome distributions were based on at least 20,000 simulations after a burn-in of at least 10,000. Treatment effects for binary outcomes were modelled using binomial likelihood and identity (VS) or logit link function (AEs and discontinuations) to estimate the risk difference and odds ratios (OR) between the treatments. Treatment effect for changes in CD4+ cell count was modelled using a normal likelihood and identity link function to estimate the difference in the mean changes from baseline to W96 between the treatments of interest. Results were expressed as the

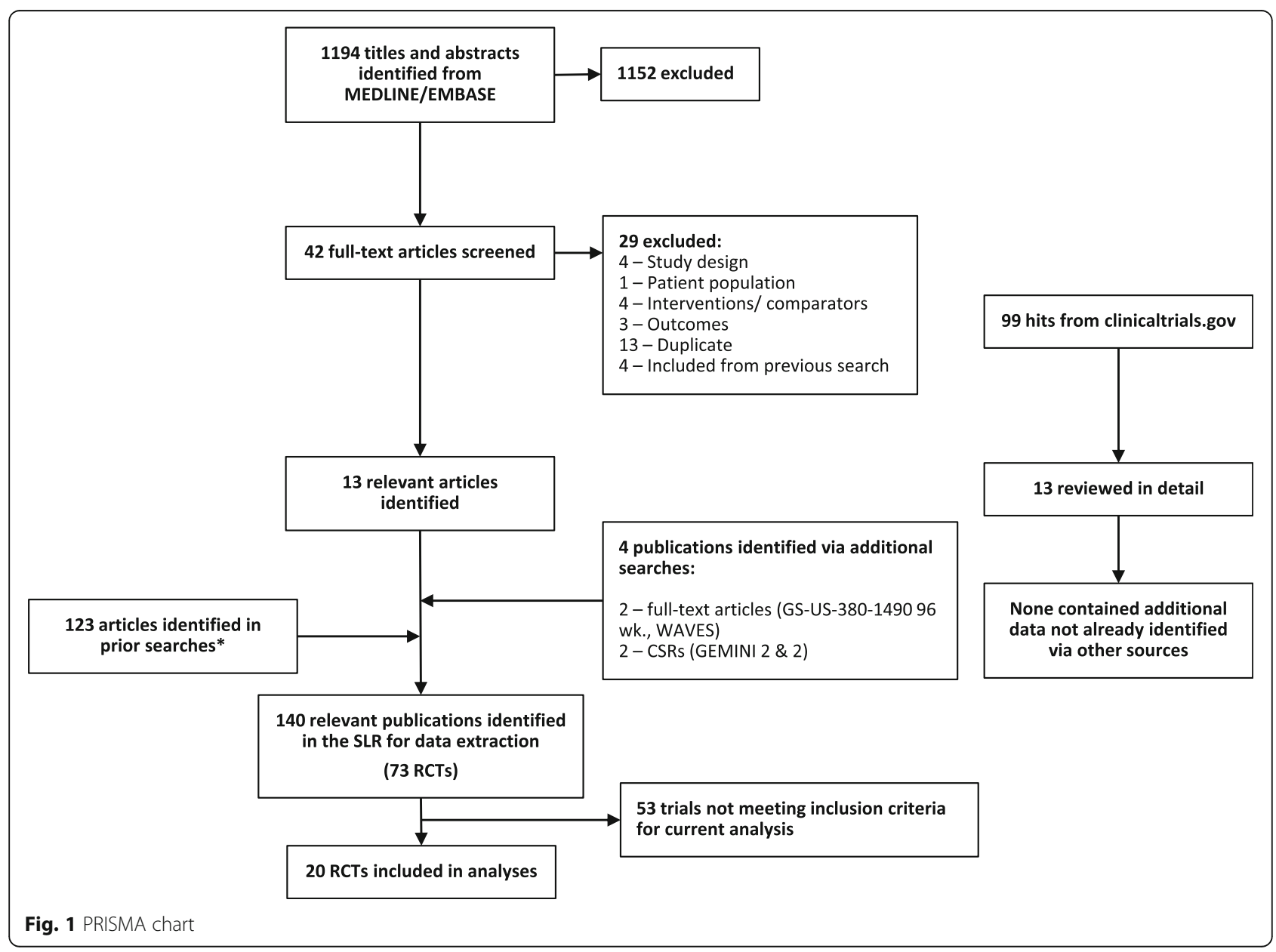


median (50th percentile) of the posterior distribution of the treatment effect and 95\% credible interval (CrI) - the 2.5th and 97.5th percentiles of the posterior distribution samples (i.e. representing the $95 \%$ probability that the parameter falls within this range). The Bayesian NMA methodology also allowed for estimates of the probability that one treatment is better than another to be calculated. As, by their nature, inferences from Bayesian analyses do not require adjustment for multiple comparisons [14], no adjustments for multiplicity were made.

\section{Results}

The systematic literature review identified a total of 1194 records from Medline and Embase databases and 100 records from clinicaltrials.gov (Fig. 1). The screening resulted in 1152 exclusions with a total of 42 full-text publications being assessed for data extraction. A further 4 records meeting the inclusion criteria were identified via secondary references. Of these, 29 articles were excluded at full-text review stage (Fig. 1). These 17 articles were added to the results of the previous SLRs, resulting in a total of 140 publications with 73 unique clinical trials [6]. Of these 20 studies conformed with the inclusion criteria and were included in the analyses [13-34].

The network of treatment comparisons for the efficacy outcomes is shown in Fig. 2. Based on model diagnostics the model with the lower DIC was used for the primary interpretation of efficacy and safety outcomes.

At W96, higher proportion of patients receiving DTG achieved VS compared to all ritonavir-boosted PIs, EFV and EVG/c, and numerically higher but not statistically significant compared to RPV, RAL and BIC (Fig. 3). RAL and BIC were statistically superior to ATV/r [\% Risk Difference (95\% Credible Interval):11.5 $(3.9,19.9)$ and 11.6 (0.5, 21.9), respectively] and LPV/r $[17.0(8.0,27.4)$ and 17.0 (5.3, 29.0), respectively], and EVG/c was superior to $\mathrm{LPV} / \mathrm{r}[11.6(0.8,23.6)]$. Among other core agents, DRV/r [11.0 (2.8, 20.1)], EFV [13.1 (3.6, 24.7)] and RPV [14.9 (3.6, 27.9)] were superior to LPV/r. The probability that treatment with DTG would result in more patients achieving and maintaining VS at W96 compared to any other core agent was greater than $80 \%$. In patients with a high viral load at baseline $(\mathrm{VL}>100,000$ copies $/ \mathrm{mL})$, a higher proportion of patients on DTG achieved VS compared to DRV/r [33.1 (13.6, 50.4)], ATV/r [38.3 (13.3, 57.7)], LPV/r [45.3 (24.1, 61.6)] and RAL [16.7 (3.3, 31.2)], and similar proportions achieved VS compared to EFV [- $0.3(-15.4$, 9.7)], RPV [2.5 (-13.7, 14.7)], EVG/c [1.6 (-18.1, 20.2)] and BIC $[6.8(-7.9,24.9)]$ at W96. EFV and BIC respectively, were superior to DRV/r [34.7 (9.3, 53.6); 25.8 (0.3, 49.3)], ATV/r [40.1 (10.2, 58.9); 30.8 (1.2, 56.0)] and LPV/ r $[47.3(20.9,61.8) ; 37.9(11.2,60.4)]$. The results in patients with $\mathrm{VL} \leq 100,000$ copies/mL were comparable between all core agents with no statistically significant differences. A total of 4 studies in the network reported data on VS among patients with baseline CD $4 \leq 200$ cells/ $\mu \mathrm{L}$ at W96, resulting in only 4 core agents: RPV, EFV, DTG and BIC in the network. Results showed that in this subgroup, the VS achieved by patients on DTG was comparable to RPV $[-0.2(-66.0,97.3)]$, EFV $[10.8(-38.4$,

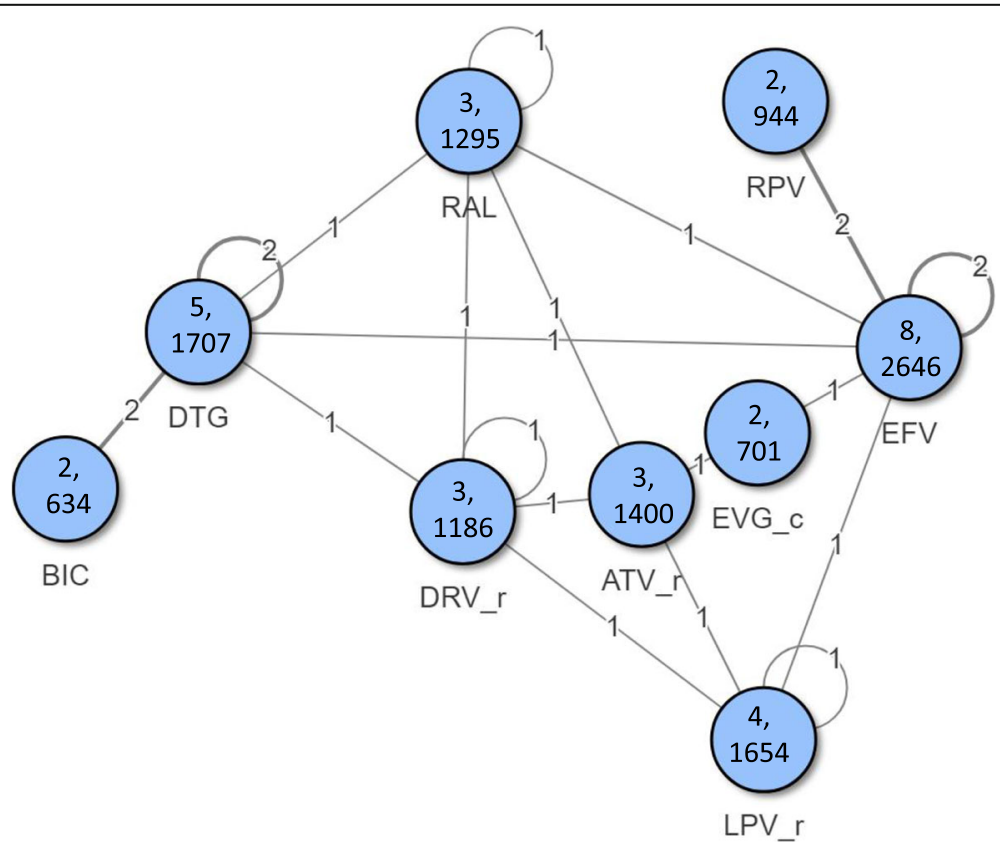

Fig. 2 Network of treatment comparisons for efficacy outcomes. *Numbers inside node represent number of studies, number of patients for each core agent 


\section{a}

DTG vs.

RE Mean Diff ( $95 \% \mathrm{Crl})$

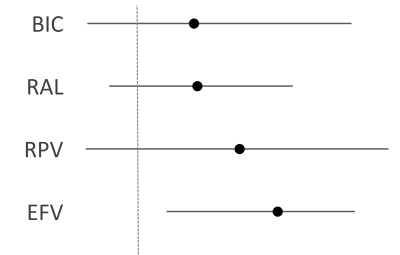

$2.7 \%(-2.7 \%, 10.6 \%)$

$2.9 \%(-1.6 \%, 7.7 \%)$

$5 \%(-2.8 \%, 12.5 \%)$

$6.9 \%(1.3 \%, 10.8 \%)$

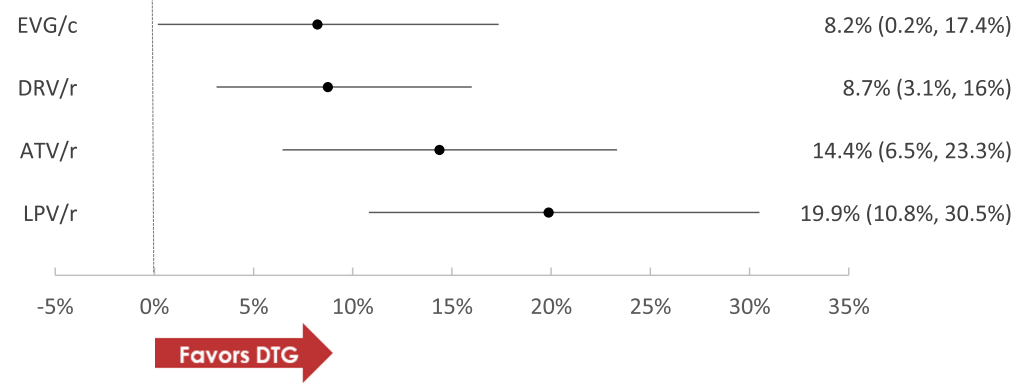

b

DTG vs.

FE Mean Diff ( $95 \% \mathrm{Crl})$

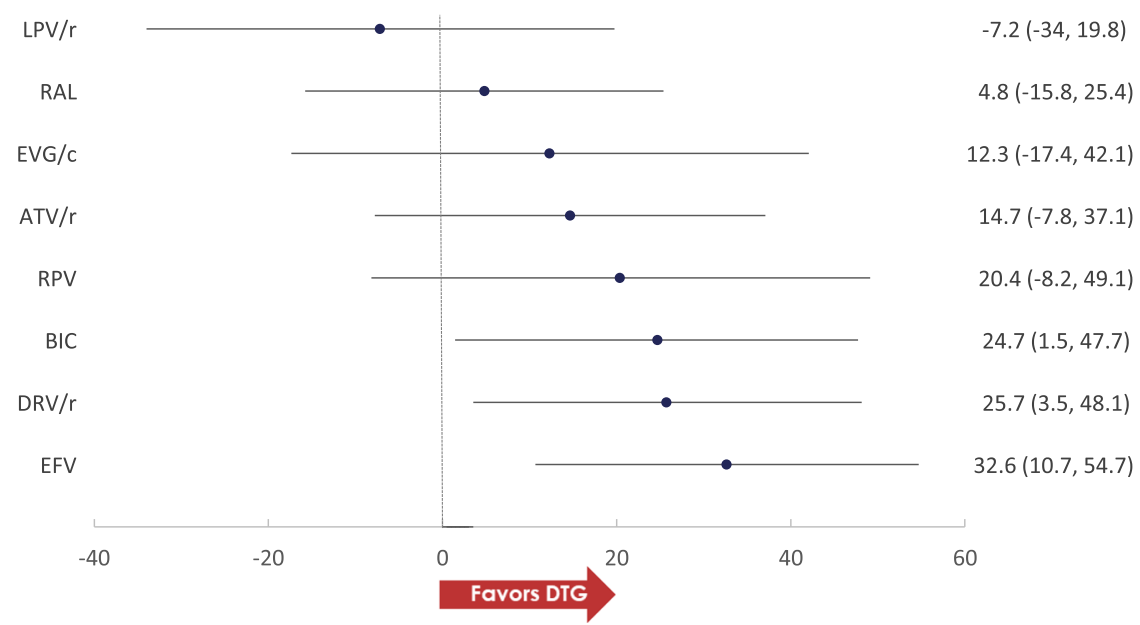

Fig. 3 Efficacy Results. A: VS Risk Difference (RE model). B: CD4 difference (FE model)

30.9)] and BIC [16.8 (-12.8, 49.3)]. Furthermore, the VS achieved by patients treated with RPV, EFV and BIC was comparable. Similarly, among patients with baseline CD4 > 200 cells $/ \mu \mathrm{L}$, the results were comparable between the four core agents with no statistically significant differences.

At W96, DTG achieved a greater increase in CD4 cell counts from baseline compared to EFV [32.6 (10.7, 54.7)], DRV/r [25.7 (3.5, 48.1)] and BIC [24.7 $(1.5,47.7)]$ and comparable changes to other core agents. Among other INSTIs, RAL achieved greater CD4 increases compared to DRV/r [20.9 (2.2, 40.0)] and EFV $[27.8(8.6,46.9)]$. The change in CD4 among patients treated with $\mathrm{LPV} / \mathrm{r}$ was greater compared with DRV/r [32.9 (11.7, 53.9)] and ATV/r [21.8 (2.4, 41.3)].

No meaningful inconsistency was observed between the direct and indirect evidence of the VS network. However, in the CD4 network, differences were observed between the estimated outcomes of the 934 [25], ABCDE [26], and GS-US-380-1489 [19] studies. These differences were found to be attributed to differential results reported for the TDF/FTC and ABC/3TC backbones. Some studies reported little difference between these backbones (HEAT [34] and ASSERT [30]), the ACTG A5202 [28] trial also reported little difference 
DTG vs.

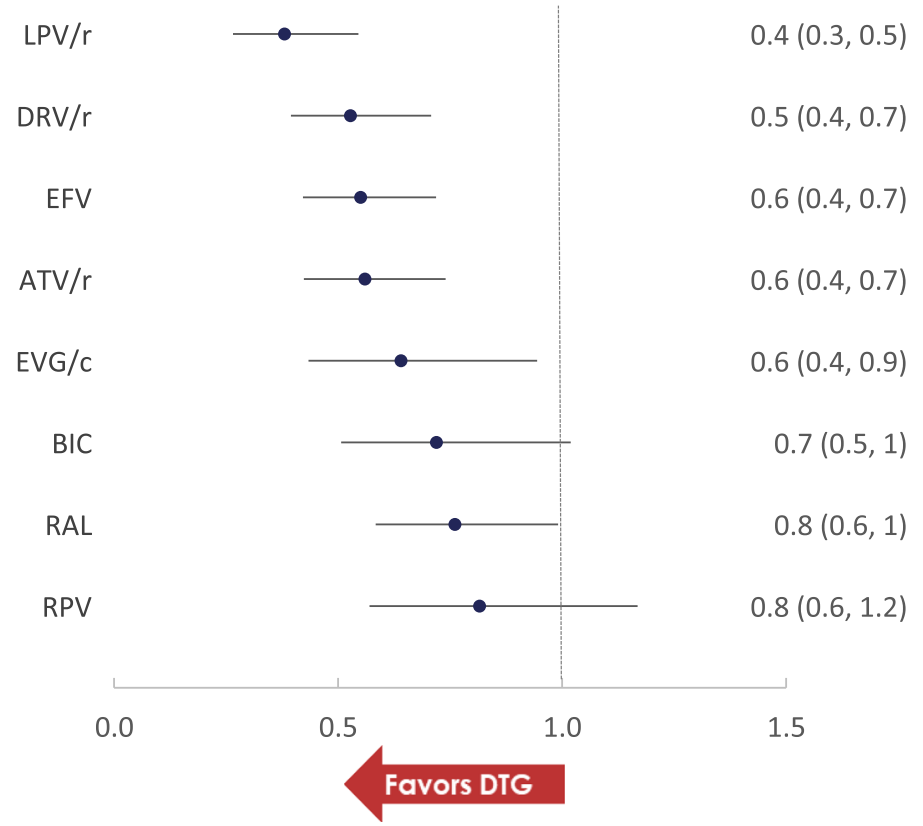

Fig. 4 Odds of all-cause discontinuation (FE model)

when combined with ATV/r, but a difference of 30 cells/ $\mu \mathrm{L}$ when combined with EFV.

\section{Safety}

Patients receiving DTG had lower odds of discontinuing therapy by W96 compared to ritonavir-boosted PIs, EFV, RAL and EVG/c (Fig. 4). The all-cause discontinuations of DTG were similar to those among patients receiving BIC or RPV. The likelihood that fewer patients receiving DTG will discontinue therapy ranged from $86.8 \%$ vs RPV to $100 \%$ vs ritonavir-boosted PI therapies. Among INSTIs, patients treated with RAL had lower odds of discontinuations compared to EFV [odds ratio (95\% CrI); $0.7(0.6,0.9)], \mathrm{DRV} / \mathrm{r}[0.7(0.5,0.9)]$, ATV/r [0.7 (0.6, 0.9)] and LPV/r $[0.5(0.4,0.7)]$. Patients treated with RPV also had lower odds of discontinuations compared to EFV $[0.7(0.5,0.9)]$, DRV/r $[0.7(0.5,0.9)]$ and $\mathrm{LPV} / \mathrm{r}[0.5(0.3,0.7)]$ whilst $\mathrm{LPV} / \mathrm{r}$ had higher odds of discontinuations compared to all core agents.

Patients on DTG had lower odds of experiencing an adverse event (AE) compared to patients on EFV [0.6 $(0.3,0.9)], \mathrm{ATV} / \mathrm{r}[0.4(0.3,0.6)]$ and LPV/r $[0.3(0.2$, $0.5)]$. The odds were comparable against other core agents such as DRV/r $[0.95(0.7,1.4)]$, RPV $[0.8(0.4$, 1.6)], RAL $[1.1(0.8,1.6)]$ and BIC $[1.2(0.8,1.8)]$. No data was available for EVG/c on AEs at week 96. Among other INSTIs, BIC and RAL, respectively had lower odds compared to EFV $[0.5(0.3,0.9) ; 0.5(0.3,0.9)]$, ATV/r $[0.3(0.2,0.6) ; 0.4(0.3,0.5)]$ and LPV/r $[0.3(0.1,0.5) ; 0.3$ $(0.2,0.4)]$. Furthermore, DRV/r had lower odds of experiencing AEs compard to ATV/r $[0.4(0.3,0.6)]$ and $\mathrm{LPV} / \mathrm{r}[0.3(0.2,0.5)]$, and RPV had lower odds compared to $\mathrm{LPV} / \mathrm{r}[0.4(0.2,1.0)]$.

Drug-related AEs were reported in 5 studies comparing 4 core agents: EFV, RAL, BIC and DTG. For patients on DTG, the odds of experiencing a drug-related $\mathrm{AE}$ were lower compared to EFV $[0.3(0.2,0.4)]$, comparable to RAL $[1.1(0.8,1.4)]$ and higher compared to BIC [1.5 $(1.1,2.0)]$. Compared to EFV, the odds of experiencing a drug-related $\mathrm{AE}$ were lower for $\mathrm{BIC}[0.2(0.1,0.3)]$ and RAL $[0.3(0.2,0.4)]$.

\section{Discussion}

INSTI-based therapies are recommended as a preferred first -line treatments for PLHIV in all major guidelines [2-4]. Previous NMAs have concluded INSTIs, specifically DTG, to have higher odds of achieving VS at W48 compared to all ritonavir-boosted PIs and NNRTIs [6, 7]. Our previous NMA also indicated that higher proportions of patients receiving DTG achieve VS compared to all core agents up to W48 [6]. Results of this analysis suggest this trend to continue up to 96 weeks. In this analysis, higher proportions of patients treated with un-boosted INSTIs (DTG, RAL and BIC) were able to achieve and maintain VS up to 96 weeks compared to $\mathrm{ATV} / \mathrm{r}, \mathrm{LPV} / \mathrm{r}, \mathrm{EFV}$ and EVG/c, with DTG being numerically better than other core agents. The changes in CD4+ cell counts from baseline were also significantly greater or comparable for patients treated with DTG compared to all other core agents suggesting DTG as a 


\section{Appendix 1}

Table 1 Study characteristics and input data for 20 trials included in the NMA

\begin{tabular}{|c|c|c|c|c|c|c|c|c|c|c|c|c|}
\hline $\begin{array}{l}\text { Study } \\
\text { (Source) }\end{array}$ & $\begin{array}{l}\text { Treatment } \\
\text { Arms }\end{array}$ & $\mathbf{N}$ & Male & $\begin{array}{l}\text { Age, } \\
y\end{array}$ & $\begin{array}{l}\text { Baseline } \\
\text { mean } \\
\text { CD4+, } \\
\text { cells/mL } \\
\text { (SD) }\end{array}$ & $\begin{array}{l}\text { Baseline mean } \\
\text { viral load, } \\
\text { log10 RNA } \\
\text { copies/mL, (SD) }\end{array}$ & $\begin{array}{l}\mathrm{N} \text { with Virologic } \\
\text { Suppression HIV } \\
\text { RNA }<50 \text { copies/ } \\
\mathrm{mL}(\mathrm{r} / \mathrm{n})\end{array}$ & $\begin{array}{l}\text { CD4+ } \\
\text { change, } \\
\text { cells/ } / \mu \mathrm{L}\end{array}$ & $\begin{array}{l}\text { AEs } \\
(r / n)\end{array}$ & $\begin{array}{l}\text { Drug- } \\
\text { related } \\
\text { AEs ( } r / \\
n)\end{array}$ & $\begin{array}{l}\text { Serious } \\
\text { AEs ( } r / \\
n)\end{array}$ & $\begin{array}{l}\text { Discontin- } \\
\text { uations } \\
(\mathrm{r} / \mathrm{n})\end{array}$ \\
\hline \multirow[t]{3}{*}{$\begin{array}{l}\text { ACTG A5257 } \\
\text { ([15]) }\end{array}$} & $\begin{array}{l}\text { ATV/r }+ \\
\text { TDF/FTC }\end{array}$ & 605 & 76.20 & 37 & 309 & $4.60^{a}$ & $379 / 605$ & 280 & $\begin{array}{l}489 / \\
605\end{array}$ & - & - & $89 / 605$ \\
\hline & $\begin{array}{l}\text { DRV/r + } \\
\text { TDF/FTC }\end{array}$ & 601 & 76.21 & 37 & 310 & $4.61^{\mathrm{a}}$ & $437 / 601$ & 256 & $\begin{array}{l}390 / \\
601\end{array}$ & - & - & $101 / 601$ \\
\hline & $\begin{array}{l}\text { RAL + TDF/ } \\
\text { FTC }\end{array}$ & 603 & 75.46 & 36 & 304 & $4.66^{\mathrm{a}}$ & $481 / 603$ & 288 & $\begin{array}{l}359 / \\
603\end{array}$ & - & - & $72 / 603$ \\
\hline \multirow[t]{2}{*}{$\begin{array}{l}\text { ECHO + } \\
\text { THRIVE [16] }\end{array}$} & $\begin{array}{l}\mathrm{EFV}+\mathrm{TDF} / \\
\mathrm{FTC}\end{array}$ & 546 & 78.94 & 36 & $261^{a}$ & $5.0^{\mathrm{a}}$ & $422 / 546$ & 222 & - & - & $61 / 546$ & - \\
\hline & $\begin{array}{l}\text { RPV + TDF/ } \\
\text { FTC }\end{array}$ & 550 & 78.00 & 36 & $247^{\mathrm{a}}$ & $5.0^{\mathrm{a}}$ & $423 / 550$ & 226 & - & - & $52 / 550$ & - \\
\hline \multirow[t]{4}{*}{$\begin{array}{l}\text { FLAMINGO } \\
\text { (CSR) [17] }\end{array}$} & $\begin{array}{l}\mathrm{DRV} / \mathrm{r}+ \\
\mathrm{ABC} / 3 \mathrm{TC}\end{array}$ & 242 & 83.06 & 34 & $400^{a}$ & $4.48^{\mathrm{a}}$ & $60 / 80$ & \multirow{2}{*}{$\begin{array}{l}274.4 \\
\text { (SD: } \\
226.64)\end{array}$} & \multirow[t]{2}{*}{$\begin{array}{l}217 / \\
242\end{array}$} & \multirow[t]{2}{*}{$124 / 242$} & \multirow[t]{2}{*}{$21 / 242$} & \multirow[t]{2}{*}{$52 / 242$} \\
\hline & $\begin{array}{l}\text { DRV/r + } \\
\text { TDF/FTC }\end{array}$ & & & & & & $104 / 162$ & & & & & \\
\hline & $\begin{array}{l}\mathrm{DTG}+\mathrm{ABC} / \\
3 \mathrm{TC}\end{array}$ & 242 & 87.19 & 34 & $390^{\mathrm{a}}$ & $4.49^{a}$ & $65 / 79$ & \multirow{2}{*}{$\begin{array}{l}298.2 \\
\text { (SD: } \\
199.95)\end{array}$} & \multirow[t]{2}{*}{$\begin{array}{l}222 / \\
242\end{array}$} & \multirow[t]{2}{*}{$83 / 242$} & \multirow[t]{2}{*}{$36 / 242$} & \multirow[t]{2}{*}{$34 / 242$} \\
\hline & $\begin{array}{l}\text { DTG + TDF/ } \\
\text { FTC }\end{array}$ & & & & & & $129 / 163$ & & & & & \\
\hline \multirow[t]{2}{*}{$\begin{array}{l}\text { GS-236-0102 } \\
{[18]}\end{array}$} & $\begin{array}{l}\mathrm{EFV}+\mathrm{TDF} / \\
\mathrm{FTC}\end{array}$ & 352 & 89.77 & 38 & $382(170.2)$ & $4.78(0.6)$ & $287 / 352$ & 273 & - & - & - & $61 / 352$ \\
\hline & $\begin{array}{l}\mathrm{EVG} / \mathrm{C}+ \\
\mathrm{TDF} / \mathrm{FTC}\end{array}$ & 348 & 88.22 & 38 & 391 (188.6) & $4.73(0.6)$ & $293 / 348$ & 295 & - & - & - & $53 / 348$ \\
\hline \multirow[t]{2}{*}{$\begin{array}{l}\text { GS-US-380- } \\
1489 \text { [19] }\end{array}$} & $\begin{array}{l}\mathrm{BIC}+\mathrm{TAF} / \\
\mathrm{FTC}\end{array}$ & 314 & 90.76 & 31 & $443^{a}$ & $4.42^{\mathrm{a}}$ & $276 / 314$ & $\begin{array}{l}287 \text { (SD: } \\
207)\end{array}$ & $\begin{array}{l}292 / \\
314\end{array}$ & $89 / 314$ & $36 / 314$ & $36 / 314$ \\
\hline & $\begin{array}{l}\text { DTG + ABC/ } \\
3 \mathrm{TC}\end{array}$ & 315 & 89.52 & 32 & $450^{\mathrm{a}}$ & $4.51^{a}$ & $283 / 315$ & $\begin{array}{l}288 \text { (SD: } \\
247)\end{array}$ & $\begin{array}{l}302 / \\
315\end{array}$ & $127 / 315$ & $39 / 315$ & $31 / 315$ \\
\hline \multirow[t]{2}{*}{$\begin{array}{l}\text { GS-US-380- } \\
1490 \text { [20] }\end{array}$} & $\begin{array}{l}\mathrm{BIC}+\mathrm{TAF} / \\
\mathrm{FTC}\end{array}$ & 320 & 87.50 & 33 & $440^{a}$ & $4.43^{\mathrm{a}}$ & $269 / 320$ & $\begin{array}{l}237 \text { (SD: } \\
204)\end{array}$ & $\begin{array}{l}283 / \\
320\end{array}$ & $64 / 320$ & $55 / 320$ & $48 / 320$ \\
\hline & $\begin{array}{l}\text { DTG + TAF/ } \\
\text { FTC }\end{array}$ & 325 & 88.62 & 34 & $441^{a}$ & $4.45^{\mathrm{a}}$ & $281 / 325$ & $\begin{array}{l}281 \text { (SD: } \\
\text { 209) }\end{array}$ & $\begin{array}{l}288 / \\
325\end{array}$ & $92 / 325$ & $33 / 325$ & $36 / 325$ \\
\hline \multirow[t]{2}{*}{$\begin{array}{l}\text { SINGLE } \\
\text { (CSR) [21] }\end{array}$} & $\begin{array}{l}\mathrm{DTG}+\mathrm{ABC} / \\
3 \mathrm{TC}\end{array}$ & 414 & 83.82 & 36 & $334.5^{\mathrm{a}}$ & $4.67^{\mathrm{a}}$ & $332 / 414$ & $\begin{array}{l}325.3 \\
(\mathrm{SE}: \\
10.46)\end{array}$ & $\begin{array}{l}376 / \\
414\end{array}$ & $184 / 414$ & $44 / 414$ & $72 / 414$ \\
\hline & $\begin{array}{l}\text { EFV + TDF/ } \\
\text { FTC }\end{array}$ & 419 & 84.96 & 35 & $339^{a}$ & $4.7^{\mathrm{a}}$ & $303 / 419$ & $\begin{array}{l}281.4 \\
(\mathrm{SE}: \\
10.87)\end{array}$ & $\begin{array}{l}394 / \\
419\end{array}$ & $282 / 419$ & $51 / 419$ & 109/419 \\
\hline \multirow[t]{4}{*}{$\begin{array}{l}\text { SPRING-2 } \\
\text { (CSR) [22] }\end{array}$} & $\begin{array}{l}\mathrm{DTG}+\mathrm{ABC} / \\
3 \mathrm{TC}\end{array}$ & 411 & 84.67 & 37 & $359^{a}$ & $4.52^{\mathrm{a}}$ & $125 / 169$ & \multirow{2}{*}{$\begin{array}{l}292.2 \\
(\mathrm{SD}: \\
195.70)\end{array}$} & \multirow[t]{2}{*}{$\begin{array}{l}349 / \\
411\end{array}$} & \multirow[t]{2}{*}{$124 / 411$} & \multirow[t]{2}{*}{$41 / 411$} & \multirow[t]{2}{*}{$62 / 411$} \\
\hline & $\begin{array}{l}\text { DTG + TDF/ } \\
\text { FTC }\end{array}$ & & & & & & $207 / 242$ & & & & & \\
\hline & $\begin{array}{l}\text { RAL + ABC/ } \\
3 \mathrm{TC}\end{array}$ & 411 & 86.37 & 35 & $362^{\mathrm{a}}$ & $4.58^{\mathrm{a}}$ & $124 / 164$ & \multirow{2}{*}{$\begin{array}{l}286.2 \\
\text { (SD: } \\
192.45)\end{array}$} & \multirow[t]{2}{*}{$\begin{array}{l}349 / \\
411\end{array}$} & \multirow[t]{2}{*}{$121 / 411$} & \multirow[t]{2}{*}{$48 / 411$} & \multirow[t]{2}{*}{$79 / 411$} \\
\hline & $\begin{array}{l}\text { RAL + TDF/ } \\
\text { FTC }\end{array}$ & & & & & & $190 / 247$ & & & & & \\
\hline \multirow{2}{*}{$\begin{array}{l}\text { STaR (GS- } \\
\text { US-264- } \\
0110) \\
{[23]}\end{array}$} & $\begin{array}{l}\mathrm{EFV}+\mathrm{TDF} / \\
\mathrm{FTC}\end{array}$ & 392 & 92.86 & 35 & 385 (187) & $4.8(0.6)$ & $284 / 392$ & $\begin{array}{l}259 \text { (SD: } \\
191)\end{array}$ & $\begin{array}{l}368 / \\
392^{b}\end{array}$ & - & - & 102/392 \\
\hline & $\begin{array}{l}\text { RPV + TDF/ } \\
\text { FTC }\end{array}$ & 394 & 92.89 & 37 & 396 (180) & $4.8(0.7)$ & $307 / 394$ & $\begin{array}{l}278 \text { (SD: } \\
189)\end{array}$ & $\begin{array}{l}362 / \\
394^{b}\end{array}$ & - & - & 78/394 \\
\hline $\begin{array}{l}\text { STARMRK } \\
\text { [24] }\end{array}$ & $\begin{array}{l}\mathrm{EFV}+\mathrm{TDF} / \\
\mathrm{FTC}\end{array}$ & 282 & 81.91 & 36.9 & $\begin{array}{l}217.4 \\
(133.6)\end{array}$ & $5(0.6)$ & $223 / 282$ & 224.8 & $\begin{array}{l}275 / \\
282\end{array}$ & $220 / 282$ & $34 / 282$ & $50 / 282$ \\
\hline
\end{tabular}


Table 1 Study characteristics and input data for 20 trials included in the NMA (Continued)

\begin{tabular}{|c|c|c|c|c|c|c|c|c|c|c|c|c|}
\hline $\begin{array}{l}\text { Study } \\
\text { (Source) }\end{array}$ & $\begin{array}{l}\text { Treatment } \\
\text { Arms }\end{array}$ & $N$ & Male & $\begin{array}{l}\text { Age, } \\
y\end{array}$ & $\begin{array}{l}\text { Baseline } \\
\text { mean } \\
\text { CD4+, } \\
\text { cells/mL } \\
\text { (SD) }\end{array}$ & $\begin{array}{l}\text { Baseline mean } \\
\text { viral load, } \\
\text { log10 RNA } \\
\text { copies/mL, (SD) }\end{array}$ & $\begin{array}{l}\text { N with Virologic } \\
\text { Suppression HIV } \\
\text { RNA }<50 \text { copies/ } \\
\mathrm{mL}(\mathrm{r} / \mathrm{n})\end{array}$ & $\begin{array}{l}\text { CD4+ } \\
\text { change, } \\
\text { cells/ } \mu \mathrm{L}\end{array}$ & $\begin{array}{l}\text { AEs } \\
(r / n)\end{array}$ & $\begin{array}{l}\text { Drug- } \\
\text { related } \\
\mathrm{AEs}(\mathrm{r} / \\
\mathrm{n})\end{array}$ & $\begin{array}{l}\text { Serious } \\
\text { AEs ( } r / \\
n)\end{array}$ & $\begin{array}{l}\text { Discontin- } \\
\text { uations } \\
(\mathrm{r} / \mathrm{n})\end{array}$ \\
\hline & $\begin{array}{l}\text { RAL + TDF/ } \\
\text { FTC }\end{array}$ & 281 & 80.78 & 37.6 & $\begin{array}{l}218.9 \\
(124.2)\end{array}$ & $5.0(0.6)$ & $228 / 281$ & 239.6 & $\begin{array}{l}266 / \\
281\end{array}$ & $132 / 281$ & $40 / 281$ & $36 / 281$ \\
\hline \multirow[t]{2}{*}{$\begin{array}{l}934 \text { study } \\
{[25]}\end{array}$} & $\begin{array}{l}\mathrm{EFV}+3 \mathrm{TC} / \\
\mathrm{ZDV}\end{array}$ & 254 & 87.01 & 37 & $241^{a}$ & $5.0^{\mathrm{a}}$ & - & $\begin{array}{l}237 \text { (SD: } \\
136.4)\end{array}$ & $\begin{array}{l}180 / \\
254\end{array}$ & - & - & - \\
\hline & $\begin{array}{l}\mathrm{EFV}+\mathrm{TDF} / \\
\mathrm{FTC}\end{array}$ & 255 & 85.88 & 36 & $233^{a}$ & $5.0^{\mathrm{a}}$ & - & $\begin{array}{l}270 \text { (SD: } \\
147.5)\end{array}$ & $\begin{array}{l}185 / \\
257\end{array}$ & - & - & - \\
\hline \multirow[t]{2}{*}{$\begin{array}{l}\text { ABCDE } \\
\text { study [26] }\end{array}$} & $\begin{array}{l}\mathrm{EFV}+\mathrm{ABC} / \\
3 \mathrm{TC}\end{array}$ & 115 & 74.8 & 38.4 & $203(167)$ & $4.94(0.60)$ & 70/115 & 263 & - & - & - & $40 / 115$ \\
\hline & $\begin{array}{l}E F V+3 T C / \\
d 4 T\end{array}$ & 122 & 78.7 & 38.5 & $223(177)$ & $4.92(0.57)$ & $58 / 122$ & 294 & - & - & - & $59 / 122$ \\
\hline \multirow[t]{2}{*}{$\begin{array}{l}\text { ACTG A5142 } \\
{[27]}\end{array}$} & $\begin{array}{l}\text { EFV + } \\
\text { CHOICE }\end{array}$ & 250 & 81.20 & 39 & 195 & 4.8 & 158/178 & 230 & - & - & - & - \\
\hline & $\begin{array}{l}\text { LPV/r + } \\
\text { CHOICE }\end{array}$ & 253 & 76.68 & 37 & 190 & 4.8 & 136/177 & 287 & - & - & - & - \\
\hline \multirow{4}{*}{$\begin{array}{l}\text { ACTG A5202 } \\
\text { [28] } \\
\text { (Clinicaltrials. } \\
\text { gov) }\end{array}$} & $\begin{array}{l}\text { ATV/r }+ \\
\text { ABC/3TC }\end{array}$ & 463 & 83.80 & 38 & $236^{a}$ & $4.7(0.7)$ & - & $250.3^{a}$ & - & - & - & $141 / 463$ \\
\hline & $\begin{array}{l}\text { ATV/r }+ \\
\text { TDF/FTC }\end{array}$ & 465 & 83.23 & 38.9 & $224^{a}$ & $4.7(0.7)$ & - & $251.5^{a}$ & - & - & - & $123 / 465$ \\
\hline & $\begin{array}{l}\mathrm{EFV}+\mathrm{ABC} / \\
3 \mathrm{TC}\end{array}$ & 465 & 78.92 & 38.4 & $225^{\mathrm{a}}$ & $4.7(0.7)$ & - & $250.5^{a}$ & - & - & - & $141 / 465$ \\
\hline & $\begin{array}{l}\text { EFV + TDF/ } \\
\text { FTC }\end{array}$ & 464 & 84.70 & 38.2 & $234^{a}$ & $4.7(0.7)$ & - & $220.5^{a}$ & - & - & - & $121 / 464$ \\
\hline \multirow[t]{2}{*}{$\begin{array}{l}\text { ARTEMIS } \\
\text { [29] }\end{array}$} & $\begin{array}{l}\text { DRV/r }+ \\
\text { TDF/FTC }\end{array}$ & 343 & 69.68 & 36 & $228^{a}$ & $7.1^{\mathrm{a}}$ & $271 / 343$ & $171^{\mathrm{a}}$ & - & - & $34 / 343$ & $59 / 343$ \\
\hline & $\begin{array}{l}\text { LPV/r+ } \\
\text { TDF/FTC }\end{array}$ & 346 & 69.65 & 35 & $218^{\mathrm{a}}$ & $6.2^{\mathrm{a}}$ & $246 / 346$ & $188^{\mathrm{a}}$ & - & - & $55 / 346$ & $81 / 346$ \\
\hline \multirow[t]{2}{*}{ ASSERT [30] } & $\begin{array}{l}\mathrm{EFV}+\mathrm{TDF} / \\
\mathrm{FTC}\end{array}$ & 193 & 79.79 & 36 & $230^{a}$ & $5.12^{\mathrm{a}}$ & $76 / 128$ & $220^{\mathrm{a}}$ & - & - & - & $59 / 193$ \\
\hline & $\begin{array}{l}\mathrm{EFV}+\mathrm{ABC} / \\
3 \mathrm{TC}\end{array}$ & 192 & 82.81 & 38 & $240^{a}$ & $5.01^{\mathrm{a}}$ & $57 / 112$ & $235^{a}$ & - & - & - & 77/192 \\
\hline \multirow[t]{2}{*}{$\begin{array}{l}\text { ATADAR } \\
{[31]}\end{array}$} & $\begin{array}{l}\text { ATV/r }+ \\
\text { TDF/FTC }\end{array}$ & 90 & 86.67 & 35 & $328(205)$ & $4.8(0.7)$ & - & $\begin{array}{l}284 \text { (SD: } \\
219)\end{array}$ & - & - & $24 / 90$ & - \\
\hline & $\begin{array}{l}\text { DRV/r }+ \\
\text { TDF/FTC }\end{array}$ & 88 & 88.64 & 37 & 341 (171) & $4.8(0.8)$ & - & $\begin{array}{l}298 \text { (SD: } \\
182)\end{array}$ & - & - & $7 / 88$ & - \\
\hline \multirow[t]{2}{*}{ CASTLE [32] } & $\begin{array}{l}\text { ATV/r }+ \\
\text { TDF/FTC }\end{array}$ & 440 & 68.64 & 34 & 205 & 5.01 & $308 / 440$ & 268 & $\begin{array}{l}355 / \\
441\end{array}$ & - & $61 / 441$ & $72 / 438$ \\
\hline & $\begin{array}{l}\text { LPV/r+ } \\
\text { TDF/FTC }\end{array}$ & 443 & 68.62 & 36 & 204 & 4.96 & $281 / 443$ & 290 & $\begin{array}{l}370 / \\
437\end{array}$ & - & $48 / 437$ & $95 / 440$ \\
\hline \multirow[t]{2}{*}{$\begin{array}{l}\text { GS-236-0103 } \\
\text { [33] }\end{array}$} & $\begin{array}{l}\text { ATV/r }+ \\
\text { TDF/FTC }\end{array}$ & 355 & 89.01 & 39 & $366^{a}$ & $4.8(0.62)$ & $292 / 355$ & 261 & - & - & $50 / 355$ & $55 / 355$ \\
\hline & $\begin{array}{l}\text { EVG/C+ } \\
\text { TDF/FTC }\end{array}$ & 353 & 91.78 & 38 & $351^{a}$ & $4.8(0.61)$ & $294 / 353$ & 256 & - & - & $34 / 353$ & $49 / 353$ \\
\hline \multirow{2}{*}{$\begin{array}{l}\text { HEAT ([34], } \\
\text { clinicaltrials. } \\
\text { gov) }\end{array}$} & $\begin{array}{l}\mathrm{LPV} / \mathrm{r}+ \\
\mathrm{ABC} / 3 \mathrm{TC}\end{array}$ & 343 & 83.97 & 38 & $214^{\mathrm{a}}$ & $4.903^{\mathrm{a}}$ & $206 / 343$ & $250^{\mathrm{a}}$ & $\begin{array}{l}274 / \\
343\end{array}$ & - & $42 / 343$ & $109 / 343$ \\
\hline & $\begin{array}{l}\text { LPV/r }+ \\
\text { TDF/FTC }\end{array}$ & 345 & 80.00 & 38 & $193^{\mathrm{a}}$ & $4.844^{\mathrm{a}}$ & $200 / 345$ & $246.5^{\mathrm{a}}$ & $\begin{array}{l}276 / \\
345\end{array}$ & - & $45 / 345$ & $124 / 345$ \\
\hline
\end{tabular}

\footnotetext{
a Median was used instead of mean

${ }^{b}$ Reported as treatment emergent adverse events, assumed to be equivalent to other reported AEs
} 
core agent to be efficacious and durable. This was supported by consistent results in difficult to treat patients with high baseline viral load or low CD4+ counts. Furthermore, these benefits were achieved without any observed risk, such as discontinuations or AEs. These data suggest that INSTIs maintain better outcomes up to 96 weeks, with DTG being the most efficacious core agents available.

DTG is the most widely used ARV globally and is already recommended in guidelines as a 2- or 3-drug combination for treatment-naïve PLHIV. A recent NMA has established comparability of DTG + 3TC combination with guideline-recommended 3-drug regimen up to 48 weeks [35]. Despite this, there have been questions about the long-term efficacy of its effect, especially in the context of a 2-drug combination. Whilst we did not compare core agents as 2-drug combinations, our results suggest that DTG, as a core agent, provides a better platform compared to any other core agent to support a 2-drug combination. A DTG-based 2-drug regimen offers a realistic alternative for patients who want to reduce their ARV exposure. Additional long-term data on DTG-based 2DRs are needed to validate this hypothesis.

Evaluation of the quality of included studies was conducted previously for 48-week analyses where all trials were found to be of "strong" or "moderate" quality [6]. Most trials with "moderate" ratings were due to the common practice of unblinded treatments in this indication. Two trials are included in this analysis that were not evaluated previously $[15,26]$, both of which were moderate quality. Similarly, the quality of the NMA comparisons evaluated by the Grading of Recommendations Assessment, Development and Evaluation approach - although not repeated for the W96 data collection - are not expected to differ from that previously reported [36].

Consistent with our previous analyses, we combined data where any core agent was used in combination with TDF- or TAF-based NRTI-backbone. This was also essential to build the network with limited number of studies reporting data up to W96. This assumption of equivalence between TDF and TAF could be perceived as a limitation of these analyses. A previous study has found TAF to be non-inferior to TDF (both with EVG/c and FTC) in terms of VS, with similar safety profiles when compared in treatment-naïve patients with HIV-1 [37].

\section{Conclusion}

Our systematic literature review and NMA provide further evidence to support the efficacy, safety and durability of INSTIs as the superior class of core agent for treatment-naïve patients with HIV-1 infection. It further reinforces DTG to be among the most durable first-line core agents up to W96, especially among difficult-totreat patients, displaying a similar safety profile. DTG can be considered as a core agent of choice when considering reducing the treatment burden for appropriate patients.

\section{Abbreviations \\ ABC/3TC: Abacavir/lamivudine; AE: Adverse event; AIDS: Acquired immunodeficiency syndrome; ART: Antiretroviral therapy; ATV/r: Ritonavir- boosted atazanavir; BIC: Bictegravir; $\mathrm{CD}^{+}$: Cluster of differentiation 4; CVR- 50: Confirmed virologic response-50; DIC: Deviance Information \\ CriterionDTGDolutegravir; DRV/r: Ritonavir-boosted darunavir; EFV: Efavirenz; EVG/c: Cobicistat-boosted elvitegravir; FE: Fixed-effect; HIV-1: Human Immunodeficiency Virus type 1; INSTIs: Integrase strand inhibitors; LPV/ r: Ritonavir-boosted lopinavir; NCT: National Institute of Health clinical trial; NMA: Network Meta-Analysis; NNRTIs: Non-nucleoside reverse transcriptase inhibitors; NRTIs: Nucleoside/nucleotide reverse transcriptase inhibitors; OR: Odds ratio; PRISMA: Preferred Reporting Items for Systematic Reviews and Meta-Analyses; Pl/rs: Ritonavir-boosted protease inhibitors; PLHIV: People living with HIV; RAL: Raltegravir; RCTs: Randomised Controlled Trials; RE: Random-effect; RPV: Rilpivirine; TD[A]F/FTC: Tenofovir disoproxil (or alafenamide) fumarate/emtricitabine; TLOVR-50: Time to loss of virologic response-50; VS: Virologic suppression; W48: 48 weeks; W96: 96 weeks}

\section{Authors' contributions}

All authors contributed to the study design. KN, NJAH and SJS contributed to data acquisition and analysis. KN, SJS and YSP contributed to results interpretation. All authors contributed to the final manuscript.

\section{Funding}

This study was funded in full by ViiV Healthcare. Manuscript writing was also funded by ViiV Healthcare. Study sponsor did not contribute to the design of the study or in the collection, analysis and interpretation of data, or in the writing of the manuscript.

\section{Availability of data and materials}

The datasets used and/or analysed during the current study are available from the corresponding author on reasonable request.

\section{Ethics approval and consent to participate}

This study was a meta-analysis of published data. Therefore, ethics approval and consent to participate were deemed not applicable.

\section{Consent for publication}

Not applicable.

\section{Competing interests}

$\mathrm{KN}, \mathrm{NJAH}$ and SJS are employees of Pharmerit International and were contracted to conduct this study on behalf of ViiV Healthcare. YSP is an employee of ViV Healthcare and holds stock in GlaxoSmithKline.

\section{Author details}

${ }^{1}$ Pharmerit International, Berlin, Germany. ${ }^{2}$ Pharmerit International, Rotterdam, Netherlands. ${ }^{3}$ Pharmerit International, Bethesda, MD, USA. ${ }^{4}$ ViiV Healthcare, GSK House, 980 Great West Rd, Brentford, Middlesex TW8 9GS, UK.

Received: 30 October 2020 Accepted: 28 January 2021

Published online: 26 February 2021

\section{References}

1. AntiretroviralTherapy Cohort Collaboration. Survival of HIV-positive patientsstarting antiretroviral therapy between 1996 and 2013: acollaborative analysis of cohort studies. Lancet HIV.2017;4(8):e349-56. https://doi.org/10.1016/S2352-3018(17)30066-8. Epub 2017 May 10.

2. DHHS Panel on Antiretroviral Guidelines for Adults and Adolescents - A Working Group of the Office of AIDS Research Advisory Council (OARAC). Guidelines for the use of antiretroviral agents in adults and adolescents 
living with HIV (updated December 2019). https://files.aidsinfo.nih.gov/ contentfiles/lvguidelines/AdultandAdolescentGL.pdf. Accessed 02 Apr 2020

3. European AIDS Clinical Society. Guidelines. Version 10.0. European AIDS Clinical Society: Brussels, Belgium; 2019.

4. WHO 2018. Updated recommendations on first-line and second-line antiretroviral regimens and post-exposure prophylaxis and recommendations on early infant diagnosis of HIV: interim guidelines. Supplement to the 2016 consolidated guidelines on the use of antiretroviraldrugs for treating and preventing HIV infection. Geneva: World Health Organization; 2018. (WHO/CDS/HIV/18.51). Licence: CC BY-NC-SA 3.0 IGO

5. Chawla A, Wang C, Patton C, Murray M, Punekar Y, de Ruiter A, et al. A review of long-term toxicity of antiretroviral treatment regimens and implications for an aging population. Infect Dis Ther. 2018;7(2):183-95.

6. Snedecor SJ, Radford M, Kratochvil D, Grove R, Punekar YS. Comparative efficacy and safety of dolutegravir relative to common core agents in treatment-naive patients infected with HIV-1: a systematic review and network meta-analysis. BMC Infect Dis. 2019;19:484.

7. Kanters S, Vitoria M, Doherty M, Socias ME, Ford N, Forrest Jl, et al, Comparative efficacy and safety of first-line antiretroviral therapy for the treatment of HIV infection: a systematic review and network meta-analysis. Lancet HIV. 2016;3(11):e510-e20.

8. Patel DA, Snedecor SJ, Tang WY, Sudharshan L, Lim JW, Cuffe R, et al. 48week efficacy and safety of dolutegravir relative to commonly used third agents in treatment-naive HIV-1-infected patients: a systematic review and network meta-analysis. PLoS One. 2014;9(9):e105653.

9. Liberati A, Altman DG, Tetzlaff J, Mulrow C, Gotzsche PC, loannidis JP, et al. The PRISMA statement for reporting systematic reviews and meta-analyses of studies that evaluate healthcare interventions: explanation and elaboration. BMJ. 2009;339:b2700.

10. U.S. Department of Health and Human Services. Guidance for Industry: Human Immunodeficiency Virus-1 Infection: Developing Antiretroviral Drugs for Treatment. 2015. http://www.fda.gov/downloads/Drugs/ GuidanceComplianceRegulatoryInformation/Guidances/UCM355128.pdf. Accessed on 21 Sept 2020.

11. Lu G, Ades AE. Combination of direct and indirect evidence in mixed treatment comparisons. Stat Med. 2004;23(20):3105-24.

12. Lunn D, Thomas A, Best N, Spiegelhalter D. WinBUGS - A Bayesian modeling framework: concepts, structure and extensibility. Stat Comput. 2000;10:325337. https://doi.org/10.1023/A:1008929526011.

13. Dias S, Welton NJ, Sutton AJ, Caldwell DM, Lu G, Ades AE. Evidence synthesis for decision making 4: inconsistency in networks of evidence based on randomized controlled trials. Med Decis Mak. 2013;33(5):641-56.

14. Gelman A, Hill J, Yajima M. Why we (usually) Don't have to worry about multiple comparisons. J Res Educ Effect. 2012;5:189-211.

15. Lennox JL, Landovitz RJ, Ribaudo HJ, et al. Efficacy and tolerability of 3 nonnucleoside reverse transcriptase inhibitor-sparing antiretroviral regimens for treatment-naive volunteers infected with HIV-1: a randomized, controlled equivalence trial. Ann Intern Med. 2014;161(7):461-71.

16. Nelson MR, Elion RA, Cohen CJ, et al. Rilpivirine versus efavirenz in HIV-1infected subjects receiving emtricitabine/tenofovir DF: pooled 96-week data from ECHO and THRIVE studies. HIV clinical trials. 2013;14(3):81-91.

17. FLAMINGO CSR. A phase IIIb, randomized, open-label study of the safety and efficacy of GSK1349572 (dolutegravir, DTG) 50 mg once daily compared to darunavir/ritonavir (DRV/r) $800 \mathrm{mg} / 100 \mathrm{mg}$ once daily each administered with fixed-dose dual nucleoside reverse transcriptase inhibitor therapy over 96 weeks in HIV-1 infected antiretroviral naïve adult subjects. 2013.

18. Zolopa A, Sax PE, DeJesus E, et al. A randomized double-blind comparison of coformulated elvitegravir/cobicistat/emtricitabine/tenofovir disoproxil fumarate versus efavirenz/emtricitabine/tenofovir disoproxil fumarate for initial treatment of HIV-1 infection: analysis of week 96 results. J Acquir Immune Defic Syndr. 2013;63(1):96-100.

19. Wohl DA, Yazdanpanah Y, Baumgarten A, et al. Bictegravir combined with emtricitabine and tenofovir alafenamide versus dolutegravir, abacavir, and lamivudine for initial treatment of HIV-1 infection: week 96 results from a randomised, double-blind, multicentre, phase 3, non-inferiority trial. Lancet HIV. 2019;6(6):e355-63.

20. Stellbrink HJ, Arribas JR, Stephens JL, et al. Co-formulated bictegravir, emtricitabine, and tenofovir alafenamide versus dolutegravir with emtricitabine and tenofovir alafenamide for initial treatment of HIV-1 infection: week 96 results from a randomised, double-blind, multicentre, phase 3, non-inferiority trial. Lancet HIV. 2019;6(6): e364-72.

21. SINGLE CSR. A phase III, randomized, double-blind study of the safety and efficacy of dolutegravir plus abacavir-lamivudine fixed-dose combination therapy administered once daily compared to Atripla over 96 weeks in HIV-1 infected antiretroviral therapy naive adult subjects. 2012.

22. SPRING-2 CSR. A Phase III, randomized, double blind study of the safety and efficacy of GSK1349572 $50 \mathrm{mg}$ once daily compared to raltegravir $400 \mathrm{mg}$ twice daily both administered with fixed-dose dual nucleoside reverse transcriptase inhibitor therapy over 96 weeks in HIV-1 infected antiretroviral naive adult subjects. 48 week results. 2010.

23. van Lunzen J, Antinori A, Cohen CJ, et al. Rilpivirine vs. efavirenz-based single-tablet regimens in treatment-naive adults: week 96 efficacy and safety from a randomized phase 3b study. AIDS. 2016:30(2):251-9.

24. Lennox JL, Dejesus E, Berger DS, et al. Raltegravir versus Efavirenz regimens in treatment-naive HIV-1-infected patients: 96-week efficacy, durability, subgroup, safety, and metabolic analyses. J Acquir Immune Defic Syndr. 2010;55(1):39-48.

25. Pozniak AL, Gallant JE, DeJesus E, et al. Tenofovir disoproxil fumarate, emtricitabine, and efavirenz versus fixed-dose zidovudine/lamivudine and efavirenz in antiretroviral-naive patients: virologic, immunologic, and morphologic changes--a 96-week analysis. J Acquir Immune Defic Syndr. 2006;43(5):535-40.

26. Podzamczer D, Ferrer E, Sanchez P, Gatell JM, Crespo M, Fisac C, Lonca M, Sanz J, Niubo J, Veloso S, Llibre JM, Barrufet P, Ribas MA, Merino E, Ribera E, Martínez-Lacasa J, Alonso C, Aranda M, Pulido F, Berenguer J, Delegido A, Pedreira JD, Lérida A, Rubio R, del Río L; ABCDE (Abacavir vs. d4T (stavudine) plus efavirenz) Study Team. Less lipoatrophy and better lipid profile with abacavir as compared to stavudine: 96-week results of a randomized study. J Acquir Immune Defic Syndr. 2007;44(2):139-47. https:// doi.org/10.1097/QAl.0b013e31802bf122.

27. Riddler SA, Haubrich R, DiRienzo AG, et al. Class-sparing regimens for initial treatment of HIV-1 infection. N Engl J Med. 2008;358(20):2095-106.

28. ACTG A5202 NCT. Efavirenz or Atazanavir/Ritonavir Given With Emtricitabine/Tenofovir Disoproxil Fumarate or Abacavir/Lamivudine in HIV Infected Treatment-Naive Adults. 2007. http://clinicaltrials.gov/ct2/show/ NCT00118898.

29. Mills AM, Nelson M, Jayaweera D, et al. Once-daily darunavir/ritonavir vs. lopinavir/ritonavir in treatment-naive, HIV-1-infected patients: 96-week analysis. Aids. 2009;23(13):1679-88.

30. Moyle GJ, Stellbrink HJ, Compston J, et al. 96-week results of abacavir/ lamivudine versus tenofovir/emtricitabine, plus efavirenz, in antiretroviral-naive, HIV-1-infected adults: ASSERT study. Antivir Ther. 2013;18(7):905-13.

31. Martinez E, Gonzalez-Cordon A, Ferrer E, et al. Differential body composition effects of protease inhibitors recommended for initial treatment of HIV infection: a randomized clinical trial. Clin Infect Dis. 2015;60(5):811-20.

32. Molina JM, Andrade-Villanueva J, Echevarria J, et al. Once-daily atazanavir/ritonavir compared with twice-daily lopinavir/ritonavir, each in combination with tenofovir and emtricitabine, for management of antiretroviral-naive HIV-1-infected patients: 96-week efficacy and safety results of the CASTLE study. J Acquir Immune Defic Syndr. 2010;53(3): 323-32.

33. Rockstroh JK, DeJesus E, Henry K, Molina JM, Gathe J, Ramanathan S, Wei X, Plummer A, Abram M, Cheng AK, Fordyce MW, Szwarcberg J; GS-236-0103 Study Team. A randomized, double-blind comparison of coformulated elvitegravir/cobicistat/emtricitabine/tenofovir DF vs ritonavir-boosted atazanavir plus coformulated emtricitabine and tenofovir DF for initial treatment of HIV-1 infection: analysis of week 96 results. J Acquir Immune Defic Syndr. 2013;62(5):483-6. https://doi.org/10.1097/QAI. Ob013e318286415c.

34. HEAT NCT. Abacavir/lamivudine versus Emtricitabine/Tenofovir both in combination with Lopinavir/ritonavir for the treatment of HIV (HEAT). 2005. http://clinicaltrials.gov/ct2/show/NCT00244712

35. Radford M, Parks DC, Ferrante S, Punekar Y. Comparative efficacy and safety and dolutegravir and lamivudine in treatment naive HIV patients. AIDS. 2019;33:1739-49. 
36. Puhan MA, Schunemann HJ, Murad MH, Li T, Brignardello-Petersen R, Singh $J A$, et al. A GRADE working group approach for rating the quality of treatment effect estimates from network meta-analysis. BMJ. 2014;349: g5630.

37. Sax PE, Wohl D, Yin MT, Post F, DeJesus E, Saag M, et al. Tenofovir alafenamide versus tenofovir disoproxil fumarate, coformulated with elvitegravir, cobicistat, and emtricitabine, for initial treatment of HIV-1 infection: two randomised, double-blind, phase 3, non-inferiority trials. Lancet. 2015;385(9987):2606-15.

\section{Publisher's Note}

Springer Nature remains neutral with regard to jurisdictional claims in published maps and institutional affiliations.

Ready to submit your research? Choose BMC and benefit from:

- fast, convenient online submission

- thorough peer review by experienced researchers in your field

- rapid publication on acceptance

- support for research data, including large and complex data types

- gold Open Access which fosters wider collaboration and increased citations

- maximum visibility for your research: over $100 \mathrm{M}$ website views per year

At $\mathrm{BMC}$, research is always in progress.

Learn more biomedcentral.com/submissions 